\title{
OBJECTS INTERACTIONS IN THE INTERNET OF THINGS: ANALYSIS OF THINGS AND THEIR CHARACTERISTICS
}

\author{
Taniawati Yenny \\ Department of Computer Science \\ University of Timor
}

\begin{abstract}
The Internet of Things is a distinct approach and many meanings for it exist. It has a lot of related terminologies that are not used evenly either, which is limiting scientific experiments. This research aims to bring open details to describe the most relevant terms like objects, devices, things, resources, identities and, more precisely, the relationships and similarities between things.
\end{abstract}

Keywords - Internet of Things; services; objects; resources; identities; discovery

\section{INTRODUCTION}

Currently, there are many different views on the definition of the Internet of Things (IoT), derived from the initial concept introduced by Kevin Ashton [1] which described the informational networks which enable the search for information about objects in real-world by giving a unique ID called Electronic Product Code (EPC) that comes with a resolution technique, to a group of actuators, sensors, and independent objects interacting directly with each other [2]. Machine-to-machine (M2M) communication concept is another approach that is associated with the IoT [3]. With such many different opinions, and with the contrast that has grown around each opinion, it is expected that there are numerous efforts at describing the Internet of Things [4][5].

The more significant thing is to agree on a description of the overall opinions in order to have a common perception of the main concepts and components that form the concept of IoT. First, we need to know what are the objects (things) that are involved in the IoT? we need to know the relationship between all resources, devices, and services? Then secondly, we need to know what do we expect while we are talking about the identification, addressing, and resolution? By analyzing the different views and within all research studies and projects, we can find that all of the aforementioned terms have been used with varying purposes by different researches which means that the IoT terminology is kind of unclear and mixed up, which results in huge scientific confusion. The main purpose of this research is to reduce the uncertainty into IoT research. We also aim to identify the most frequent usage of all other concepts that used with the IoT.

\author{
Rasik Bahri \\ Department of Computer Science and Engineering \\ Kalimantan Institute of Technology
}

\section{OBJECTS AND DEVICES}

All the many definitions of the concept "IoT" have in general that it is relevant to the combination of the real world with the Internet. This means that there are real physical things we need to be able to monitor and to communicate with. For example, having a physical object like a sink, bed, automobile, machines, refrigerator, or a box of soda or cup of coffee in addition to objects like humans or animals. We can call these are the objects that are connected and used within the concept IoT [7]. Houses, shops, and things in the real environment like mountains and rivers can also be addressed. Any object that has different attributes used to describe it can be considered as an entity of things.

To track and communicate with one or more objects and apply a linkage to the Internet, we need to have network devices. These devices are linked to or built-in the things themselves - thus allowing smart objects to be placed in the real world and can be monitored.

For examples of objects containing RFID reading devices such as computers, phones actuator devices, and sensors. There are many of views that about the IoT devices as the objects used in the IoT concept, which looks too limited, as real businesses are looking for the physical thing rather than any special devices used for sending/receiving messages and for monitoring. This means that devices compose things of interest by themselves when considering them from a high management view. Therefore, these things or devices are part of all the objects in the IoT. Nonetheless, to clarify this matter where the objects are the devices and the entity of things are equivalent which has to be considered as a different case.

Things normally contain resources which can be considered as computational parts which shows the technical connection to the entities of things. For example, they contain details about the object, as an identity or read information. Furthermore, they can have sensing/actuation features. In order to communicate with these objects over the internet through web services. Objects can use application interfacing services directly in order to provide extra levels of collection and abstraction. Other services [8][9] are generally used most appropriately when directly reaching the objects, but some other applications and technologies like or Device Profile for 


\section{International Journal of Engineering Applied Sciences and Technology, 2020 \\ Vol. 4, Issue 11, ISSN No. 2455-2143, Pages 489-492 \\ Published Online March 2020 in IJEAST (http://www.ijeast.com)}

Web Services (DPWS) [10] are most likely different; in particular, there is a need for higher aggregated features that should be combined with IoT applications. On the other hand, by using REST [11], the difference between services and resource becomes more ambiguous, but it could be resolved by using the services when considering the application combination and accessing features while considering the resources when working from more lower IoT elements and deployment aspect. The connection linking all these resources is shown in Figure 1 which contains an entity of things that are tracked by an external object in the real environment, while it also can use a device embedded inside it. As we have described, all resources of objects can be described as entities of things when viewing from a high management view, which shows the relationship between them. The objects can contain one or more features which can be reached through IoT services.

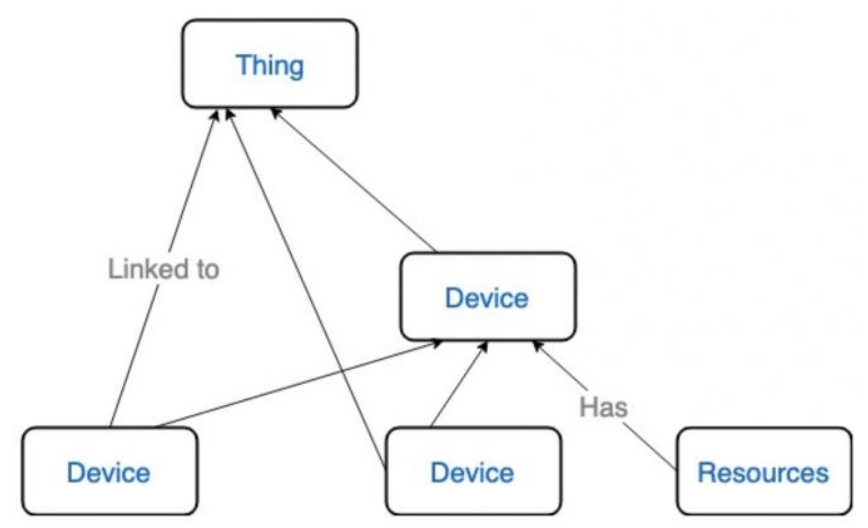

Fig. 1. The relationship among objects, devices, things, resources, and IoT services

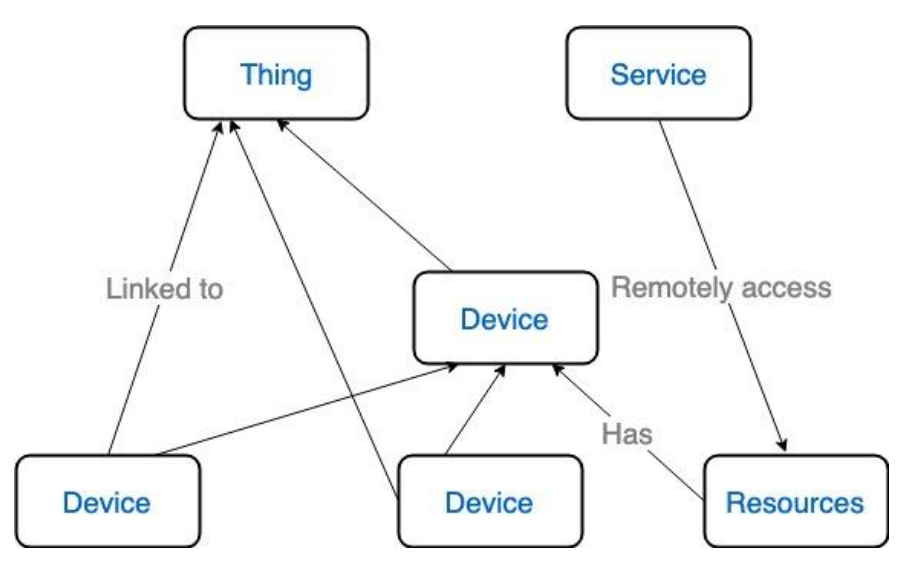

Fig. 1. The relationship among objects, devices, things, resources, and IoT services after adding remotely connected access devices

\section{EXAMPLES OF OBJECTS AND DEVICES}

The difference between entities of things and IoT devices is regularly obvious - the entity of things represent objects that have any value used for observations, while the IoT device is a special part that can track or communicate with the entity of things. However, there are more cases that the differences are more complicated which needs to be investigated. So, we are going to discuss some cases in common related areas for the Internet of Things: Logistics requirements, energy consumptions, and further IoT management, IoT safety and risk management.

We begin by considering at a box filled with some chemical that has a sensitive temperature measured from A to B. The location of the box needs to be monitored continuously, and for assuring that the quality is not affected, the temperature should be tracked to make sure that it is not exceeding the normal temperature range during all times. In this case, we need to place a WIFI sensor that is linked to the box. The WIFI sensor enables communication to the device as well as allows to record temperature reading. With this simple case, the difference is clear: the $B$ represents the entity of things, while the WIFI sensor is directly linked to the device box.

Each object has a barcode sticker which we can use to represent an entity of things, however, the barcode sticker is a major component added to the device to enable the identification and tracking features, it also qualifies to be called a device. The barcode sticker represents a device which helps in the entity of things. We can consider the barcode sticker as a single device, but this could open a different understanding of these representations. For this reason, we need to replace the sticker that contains the barcode with an RFID chip: The chip can easily enables communications with the device, and in its storage, it contains an identification number and extra device data. RFID system can work as the barcode sticker scanner by passing their monitoring reading data. With the energy monitoring, we can add reading meters to track the usage of electricity remotely and in smart grid scenarios, also distributed electricity production - are one of the key components to minimize energy usage and allowing an accurate billing based on individual consumption. In more and more countries the usage of smart meters is mandated [12]. They also represent a good example of this work. They can be a device and representing an entity of things at the same time. When considering their design, they are simply an electronic device that tracks energy usage. However, they are more complicated to represent an entity of things.

Some energy control systems, smart devices are installed to measure the demand for the energy to help them to prevent reaching the highest load. For instance, a fridge freezer may be switched off for a specific time without affecting the stored containers inside it. This example shows a simple difference between the device and the entity of things. While on other 


\section{International Journal of Engineering Applied Sciences and Technology, 2020 \\ Vol. 4, Issue 11, ISSN No. 2455-2143, Pages 489-492 \\ Published Online March 2020 in IJEAST (http://www.ijeast.com)}

cases, the fridge freezer can represent the entity of things while the internal parts (the device) which can remotely provide the fridge freezer with instructions to be switched on or off.

\section{IDENTIFICATION AND ADDRESSING}

We often can find a misunderstanding of the meanings of the terms. An entity of things can have only one character, while it may come with different meanings related to it. These meanings cab always increase the uncertainty. Of course, depending on the environment, meanings can be applied.

Addressing: is a special term always used to represent the ability to communicate with a device which means here the meaning of the device is mostly similar to the address such as Internet Protocol (IP) address and the Media Access Control (MAC) address. Back to the aforementioned example, if we consider the box that is a fully filled of a chemical. Then, the box should have an ID number to be able to be tracked. We can use this ID to be stored along with the corresponding data in the storage such as location and type. The previous 'box' example of the WIFI sensor can use IP addresses for further functions and feature for the reading temperature. Then, we save the temperature measurements in database storage as part of the entity of things which can be later accessed using a unique ID number and the ID address is quickly used to perform interactions and linkage with the device.

\section{CONCLUSION}

In this research, the most significant meanings of the IoT and the relationship within these meanings have been addressed: the objects, things, resources, services, and devices. We have proved that the main difference between the entity of things and the object is a simple classification that is not always achievable. First, it is related to the view from the individual's perspective and its environment. The main purpose was to eliminate some of the uncertainty in scientific researches and the application of the IoT as the main factor of future technology. The potential application of our definition in the future can guarantee that related research and application on the area of the IoT can improve more efficiently. Having a precise meaning will enable concentrating on the real IoT research and issues that are related to communications and interactions, management, and how to form business methods that communicate with things in the real physical world. Simply by addressing these concerns, it will enhance more advantages and benefits to the IoT that have been introduced.

\section{REFERENCE}

[1] Da Xu, L., He, W., \& Li, S. (2014). Internet of things in industries: A survey. IEEE Transactions on industrial informatics, 10(4), 2233-2243.
[2] Atzori, L., Iera, A., \& Morabito, G. (2010). The internet of things: A survey. Computer networks, 54(15), 27872805.

[3] Alharbi, S., \& Drew, S. (2018, November). The Role of Self-efficacy in Technology Acceptance. In Proceedings of the Future Technologies Conference (pp. 1142-1150). Springer, Cham.

[4] Alharbi, S., \& Drew, S. (2014, August). Mobile learningsystem usage: An integrated framework to measure students' behavioural intention. In 2014 Science and Information Conference (pp. 906-911). IEEE.

[5] AlZubi, A. A., Al-Maitah, M., \& Alarifi, A. (2019). A best-fit routing algorithm for non-redundant communication in large-scale IoT based network. Computer Networks, 152, 106-113.

[6] Al-Saggaf, U. M., Mehedi, I. M., Mansouri, R., \& Bettayeb, M. (2016). State feedback with fractional integral control design based on the Bode's ideal transfer function. International Journal of Systems Science, 47(1), 149-161.

[7] Almohaimeed, A., \& Asaduzzaman, A. (2019, January). Introducing Edge Controlling to Software Defined Networking to Reduce Processing Time. In 2019 IEEE 9th Annual Computing and Communication Workshop and Conference (CCWC) (pp. 0585-0590). IEEE.

[8] Alelyani, S., Tang, J., \& Liu, H. (2018). Feature selection for clustering: A review. In Data Clustering (pp. 29-60). Chapman and Hall/CRC.

[9] Ahmed, A. A., \& Alzahrani, A. A. (2019). A comprehensive survey on handover management for vehicular ad hoc network based on $5 \mathrm{G}$ mobile networks technology. Transactions on Emerging Telecommunications Technologies, e3546.

[10] Yoshida, Y., Maruta, A., Kitayama, K. I., Nishihara, M., Tanaka, T., Takahara, T., ... \& Yan, S. (2014). SDNbased network orchestration of variable-capacity optical packet switching network over programmable flexi-grid elastic optical path network. Journal of Lightwave Technology, 33(3), 609-617.

[11] Alghamdi, F., Mahfoudh, S., \& Barnawi, A. (2019). A Novel Fog Computing Based Architecture to Improve the Performance in Content Delivery Networks. Wireless Communications and Mobile Computing, 2019.

[12] Alghamdi, F., Mahfoudh, S., \& Barnawi, A. (2019). A Novel Fog Computing Based Architecture to Improve the Performance in Content Delivery Networks. Wireless Communications and Mobile Computing, 2019.

[13] Alelyani, S., \& Ibrahim, A. (2018, February). Internet-ofthings in telemedicine for diabetes management. In 2018 15th learning and technology conference (L\&T) (pp. 2023). IEEE. 
[14] Almohaimeed, A., \& Asaduzzaman, A. (2019, March). Incorporating Monitoring Points in SDN to Ensure Trusted Links Against Misbehaving Traffic Flows. In 2019 Fifth Conference on Mobile and Secure Services (MobiSecServ)(pp. 1-4). IEEE.

[15] Al-Saggaf, U. M., Mehedi, I. M., Mansouri, R., \& Bettayeb, M. (2017). Rotary flexible joint control by fractional order controllers. International Journal of Control, Automation and Systems, 15(6), 2561-2569.

[16] Al-Fuqaha, A., Guizani, M., Mohammadi, M., Aledhari, M., \& Ayyash, M. (2015). Internet of things: A survey on enabling technologies, protocols, and applications. IEEE communications surveys \& tutorials, 17(4), 2347-2376. 